\title{
Acute Hyperglycemia Abolishes Ischemic Preconditioning by Inhibiting Akt Phosphorylation: Normalizing Blood Glucose before Ischemia Restores Ischemic Preconditioning
}

\author{
Zequan Yang, ${ }^{1,2}$ Yikui Tian, ${ }^{1}$ Yuan Liu, ${ }^{1}$ Sara Hennessy, ${ }^{1}$ \\ Irving L. Kron, ${ }^{1}$ and Brent A. French ${ }^{2}$ \\ ${ }^{1}$ Department of Surgery, University of Virginia Health System, P.O. Box 800709, Charlottesville, VA 22908, USA \\ ${ }^{2}$ Department of Biomedical Engineering, University of Virginia Health System, P.O. Box 800759, Charlottesville, VA 22908, USA
}

Correspondence should be addressed to Zequan Yang; zy6b@virginia.edu

Received 17 August 2013; Accepted 5 November 2013

Academic Editor: Qian Fan

Copyright (C) 2013 Zequan Yang et al. This is an open access article distributed under the Creative Commons Attribution License, which permits unrestricted use, distribution, and reproduction in any medium, provided the original work is properly cited.

\begin{abstract}
This study examined the hypothesis that acute hyperglycemia (HG) blocks ischemic preconditioning (IPC) by inhibiting Akt phosphorylation. Brief HG of approximately $400 \mathrm{mg} / \mathrm{dL}$ was induced in C57BL/6 mice via intraperitoneal injection of $20 \%$ dextrose $(2 \mathrm{~g} / \mathrm{kg})$. All mice underwent $40 \mathrm{~min}$ LAD occlusion and $60 \mathrm{~min}$ reperfusion. The IPC protocol was 2 cycles of $5 \mathrm{~min}$ ischemia and 5 min reperfusion prior to index ischemia. Results. In control mice, infarct size (IF) was $51.7 \pm 2.0$ (\% risk region). Preconditioning reduced IF by $50 \%$ to $25.8 \pm 3.2(P<0.05$ versus control). In HG mice, IF was significantly exacerbated to $58.1 \pm 2.3$. However, the effect of IPC completely disappeared in HG mice. Normalization of blood glucose with insulin 5 min before IPC recovered the cardioprotective effect. Administration of CCPA before index ischemia mimicked IPC effect. The cardioprotective effect of CCPA, not its chronotropic effect, completely disappeared in HG mice. Phosphorylation of cardiac tissue Akt before index ischemia was enhanced by IPC or CCPA but was significantly inhibited by HG in both groups. Normalization of glucose with insulin reversed the inhibition of Akt phosphorylation by HG. Conclusion. HG abolishes the cardioprotective effect of preconditioning by inhibiting Akt phosphorylation. Normalization of blood glucose with insulin suffices to recover the cardioprotective effect of preconditioning.
\end{abstract}

\section{Introduction}

Hyperglycemia is commonly present in the perioperative period in patients undergoing cardiac surgery [1-3]. Hyperglycemia during cardiopulmonary bypass is an independent risk factor for mortality and morbidity in both diabetic and nondiabetic patients [3]. An increasing body of clinical evidence has shown that acute hyperglycemia (or stress hyperglycemia) is independently associated with larger myocardial infarct (MI) size and impaired left ventricular function in both diabetic and nondiabetic patients [4-6]. Animal studies have also shown that the size of MI increases in response to elevations in blood glucose levels $[7,8]$.

Ischemic preconditioning is a powerful endogenous protective mechanism against myocardial ischemia/reperfusion injury, which is induced by brief episodes of ischemia and reperfusion before the prolonged index myocardial ischemia and reperfusion [9]. However, diabetes mellitus and acute hyperglycemia have been shown to counteract the cardioprotective effects of both ischemic and pharmacological preconditioning in animals and humans [7, 10-12]. The mechanisms underlying the hyperglycemic blockade of preconditioning remain to be defined. Insulin has been used to treat acute or stress hyperglycemia clinically. It is also well known that insulin exerts a salutary preconditioning effect against myocardial ischemia/reperfusion injury $[13,14]$. In the setting of hyperglycemia, only a few conflicting results have been reported on the effect of preconditioning-mimetic insulin. Animal studies have reported that the detrimental effects of acute hyperglycemia on the exacerbation of myocardial 
infarction or the blockade of ischemic preconditioning are independent of insulin $[7,15]$. However, other studies seem to favor the use of insulin to restore the preconditioning effect in hyperglycemic patients or animals $[16,17]$. It is now well known that activation of adenosine $A_{1}$ receptors $\left(A_{1} R\right)$, either by ischemic preconditioning or by specific agonists, triggers the protective effect against myocardial ischemia/reperfusion injury [18-24]. However, the role of acute hyperglycemia in blocking the $A_{1} R$ pathway is largely unknown.

The current study employed an in vivo mouse model of myocardial ischemia and reperfusion injury to evaluate $\mathrm{A}_{1} \mathrm{R}$ signaling and Akt phosphorylation in the hyperglycemic inhibition of ischemic preconditioning, as well as to evaluate the role of insulin in restoring the effect of ischemic preconditioning in mice with acute hyperglycemia.

\section{Materials and Methods}

This study conformed to the Guide for the Care and Use of Laboratory Animals published by the National Institutes of Health (Eighth Edition, revised 2011) and was conducted under protocols approved by the University of Virginia's Institutional Animal Care and Use Committee.

2.1. Agents and Chemicals. Triphenyl tetrazolium chloride (TTC) and 2-chloro-N(6)-cyclopentyladenosine (CCPA) were purchased from Sigma-Aldrich (St. Louis, MO). Phthalo blue was purchased from Heucotech Ltd. (Fairless Hills, PA). Clinical-grade insulin was purchased from Eli Lily (Indianapolis, IN).

2.2. Animals and Experimental Protocol. Seventy-two C57BL/ 6 mice (9-13 weeks old) were purchased from Jackson Laboratories for use in this study. Three mice died and three mice were excluded due to technical failures in Phthalo blue staining. The rest of the mice, total of 66 , were assigned to 8 different groups that underwent 40 minutes of ischemia and 60 minutes of reperfusion as shown in Figure 1. An additional 4 mice/group representing each of these 8 groups were treated similarly and euthanized to provide heart tissue before index ischemia.

Acute hyperglycemia was induced by i.p. injection of $20 \%$ dextrose 50 minutes prior to LAD occlusion at a dose of $2 \mu \mathrm{L} / \mathrm{g}$ body weight [8]. Ischemic preconditioning was applied to mice 30 minutes after saline or glucose injection with two cycles of 5 minutes ischemia and 5 minutes reperfusion. In insulin-treated mice, the insulin was injected via external jugular vein 10 minutes before the ischemic preconditioning or before index LAD occlusion (nonpreconditioned mice) at a dose of $0.2-0.4 \times 10^{-3}$ unit/g $(0.1 \mathrm{unit} / \mathrm{mL}, 2-4 \mu \mathrm{L} / \mathrm{g})$ to normalize blood glucose levels. In CCPA-treated groups, CCPA was administered 20 minutes before index LAD occlusion at a dose of $25 \mu \mathrm{g} / \mathrm{kg}$ via external jugular vein.

2.3. Myocardial Ischemia/Reperfusion Injury and Measurement of Infarct Size. Mice were subjected to 40 minutes of coronary occlusion followed by 60 minutes of reperfusion as detailed previously $[8,25,26]$. Briefly, mice were anesthetized with sodium pentobarbital (100 mg/kg i.p.) and orally intubated. Artificial respiration was maintained with a $\mathrm{FiO}_{2}$ of $0.80,100$ strokes per minute, and a 0.2 to $0.5-\mathrm{mL}$ stroke volume. The heart was exposed through a left thoracotomy. A 7-0 silk suture was placed around the LAD at a level $1 \mathrm{~mm}$ inferior to the left auricle, and a miniature balloon occluder fashioned from microbore Tygon tubing (Small Part Inc., Seattle, WA) was affixed over the LAD. Ischemia and reperfusion were induced by inflating or deflating the balloon, respectively. ECG was monitored perioperatively using PowerLab instrumentation (ADInstruments, Colorado Springs, CO). The mice were euthanized 60 minutes after reperfusion, and the hearts were cannulated through the ascending aorta for perfusion with 3 to $4 \mathrm{~mL}$ of $1.0 \%$ TTC. The LAD was then reoccluded with the same suture used for coronary occlusion, and 10\% Phthalo blue was then perfused to determine risk region (RR). The left ventricle was then cut into 5 to 7 transverse slices that were weighed and digitally photographed to determine infarct size as a percent of RR.

2.4. Western Blot. Total protein was extracted from the indicated experimental groups using RIPA buffer, and protein concentration was determined by BCA protein assay (Thermo Scientific, Rockford, IL). All western blots were performed according to standard procedures. Twenty micrograms of protein were separated by $12 \%$ SDS-PAGE. After transfer, nitrocellulose membranes (Bio-Rad, Hercules, CA) were probed with primary antibodies detecting pan-AKT or phospho-AKT S473 (Cell Signaling Technology, Beverly, MA) at a 1:1,000 dilution. Secondary antibodies (Promega, Madison, WI) were applied at a 1:5,000 dilution in blocking solution (1\% milk in TBS-T). Proteins were visualized with enhanced chemiluminescent substrate (Thermo Scientific, Rockford, IL) followed by densitometry analysis using FluorChem 8900 imaging system (Alpha Innotech, Santa Clara, CA).

2.5. Statistical Analysis. All data are presented as mean \pm SEM (standard error of the mean). Peri-ischemic heart rate changes were analyzed using repeated measures ANOVA followed by Bonferroni pairwise comparisons. All other data were compared using one-way ANOVA followed by $t$-test for unpaired data with Bonferroni correction.

\section{Results}

3.1. Perioperative Heart Rate Changes. Table 1 reports changes in heart rate before, during, and after LAD occlusion. Heart rate increased significantly after LAD occlusion and remained elevated until early reperfusion. There was no significant difference in heart rate between control and treated mice. In mice with ischemic preconditioning, heart rate also increased significantly after LAD occlusion. On the first cycle of reperfusion, up to $40 \%$ of all mice developed transient tachycardia with the heart rate reaching from 1000 to $1500 \mathrm{bpm}$. This lasted a few seconds and spontaneously returned back to baseline. The incidence of tachycardia was not different between any of the preconditioning groups. 


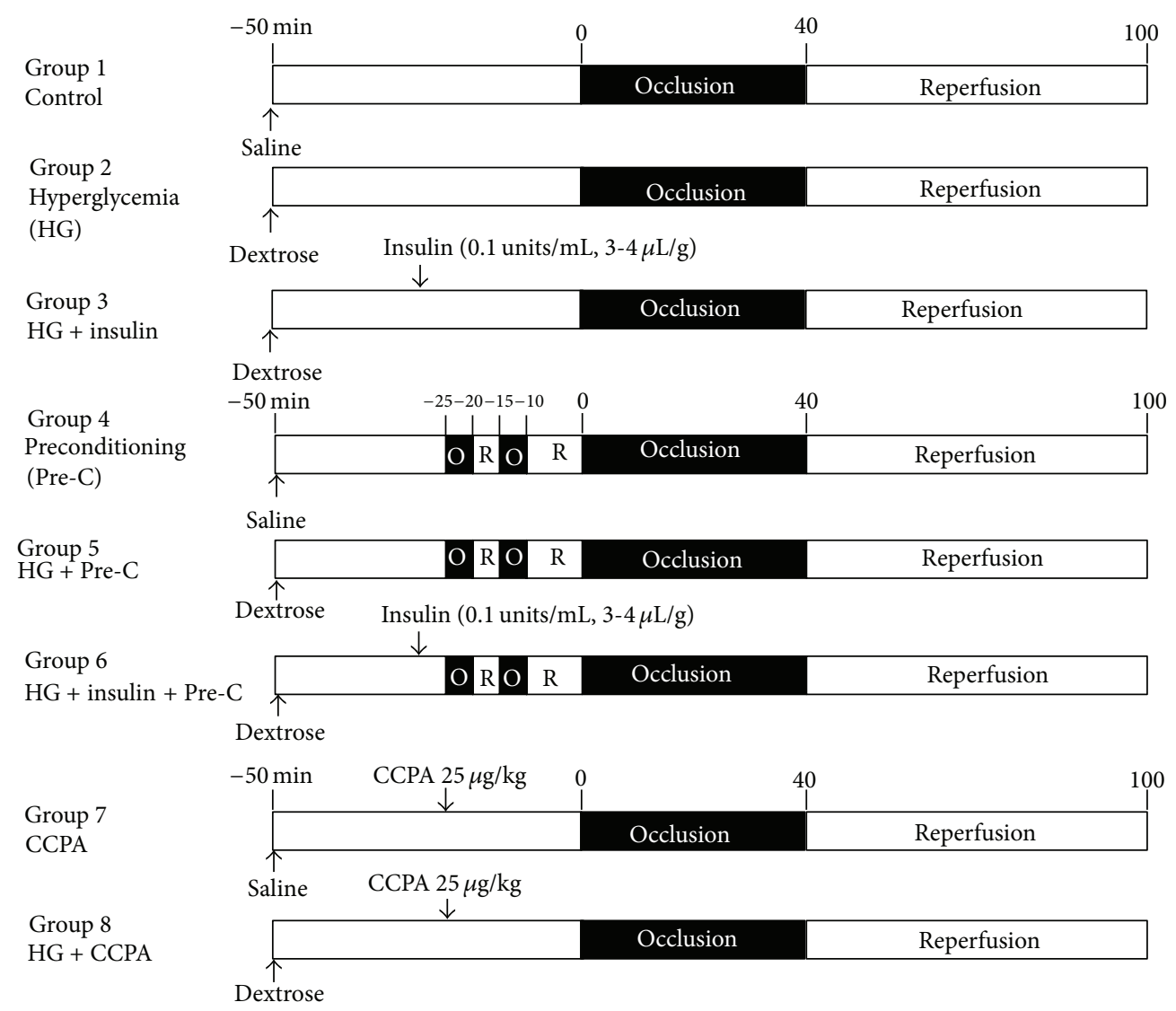

Figure 1: Animal groups and experimental protocols.

TABLE 1: Blood glucose levels before index LAD occlusion and perioperative heart rates.

\begin{tabular}{|c|c|c|c|c|c|}
\hline \multirow{2}{*}{ Groups } & \multicolumn{2}{|c|}{ Blood glucose $(\mathrm{mg} / \mathrm{dL})$} & \multicolumn{3}{|c|}{ Heart rate (beats per minutes) } \\
\hline & $5^{\prime}$ before Pre- $\mathrm{C}$ & $5^{\prime}$ before occlusion & Before occlusion & During occlusion & Reperfusion \\
\hline Control & & $168 \pm 27$ & $415 \pm 9$ & $484 \pm 9^{\#}$ & $479 \pm 11^{\#}$ \\
\hline HG & & $438 \pm 51^{*}$ & $432 \pm 10$ & $516 \pm 14^{\#}$ & $521 \pm 15^{\#}$ \\
\hline HG + insulin & $467 \pm 47^{\Pi}$ & $140 \pm 12$ & $393 \pm 7$ & $483 \pm 30^{\#}$ & $477 \pm 20^{\#}$ \\
\hline Pre-C & & $159 \pm 32$ & $394 \pm 7$ & $455 \pm 13^{\#}$ & $450 \pm 7^{\#}$ \\
\hline $\mathrm{HG}+$ Pre-C & & $454 \pm 12^{*}$ & $401 \pm 7$ & $487 \pm 17^{\#}$ & $511 \pm 15^{\#}$ \\
\hline $\mathrm{HG}+$ Pre-C + insulin & $407 \pm 169^{\Pi}$ & $125 \pm 7$ & $395 \pm 12$ & $492 \pm 29^{\#}$ & $494 \pm 20^{\#}$ \\
\hline
\end{tabular}

HG: hyperglycemia; Pre-C: ischemic preconditioning.

${ }^{*} P<0.05$ versus non-HG groups; ${ }^{\Pi} P<0.05$ versus 5 min before index LAD occlusion; ${ }^{\#} P<0.05$ versus before index LAD occlusion.

However, this type of arrhythmia was not seen after the prolonged index LAD occlusion.

3.2. Acute Hyperglycemia and Normalization by Insulin in Mice. Blood glucose levels were monitored with a conventional glucose meter (iTest, Auto Control Med. Inc., Canada) by puncturing the tail vein. A single intraperitoneal bolus injection of $20 \%$ dextrose $(10 \mu \mathrm{L} / \mathrm{g}$ or $2 \mathrm{~g}$ dextrose $/ \mathrm{kg}$ body weight) achieved transient blood glucose levels between 400 and $500 \mathrm{mg} / \mathrm{dL}$ within 20 to 30 minutes (Table 1). Intravenous injection of insulin at a dose of $0.2-0.4 \times 10^{-3}$ unit/g normalized blood glucose levels to $100-200 \mathrm{mg} / \mathrm{dL}$ within $5 \mathrm{~min}$ $(P<0.05)$.
3.3. Acute Hyperglycemia Exacerbates Myocardial Ischemial Reperfusion Injury. Three groups of mice (Figure 1, top 3 groups) underwent $40 \mathrm{~min}$ of LAD occlusion followed by $60 \mathrm{~min}$ of reperfusion. The mean blood glucose levels before LAD occlusion were $168 \pm 27 \mathrm{mg} / \mathrm{dL}$ in saline-treated control mice (control group), $438 \pm 51 \mathrm{mg} / \mathrm{dL}$ in hyperglycemic mice (HG group), and $467 \pm 47 \mathrm{mg} / \mathrm{dL}$ before insulin injection and $140 \pm 12 \mathrm{mg} / \mathrm{dL}$ after insulin treatment in HG+insulintreated mice (HG+insulin group) (see Table 1 ). There were no statistical differences in risk region ( $R R, \%$ of $L V)$ among these three groups of mice $(P>0.05$, left set of columns in Figure 2). The infarct size in control mice was $51.7 \pm 2.0(\%$ of RR). In HG mice, infarct size was significantly exacerbated 


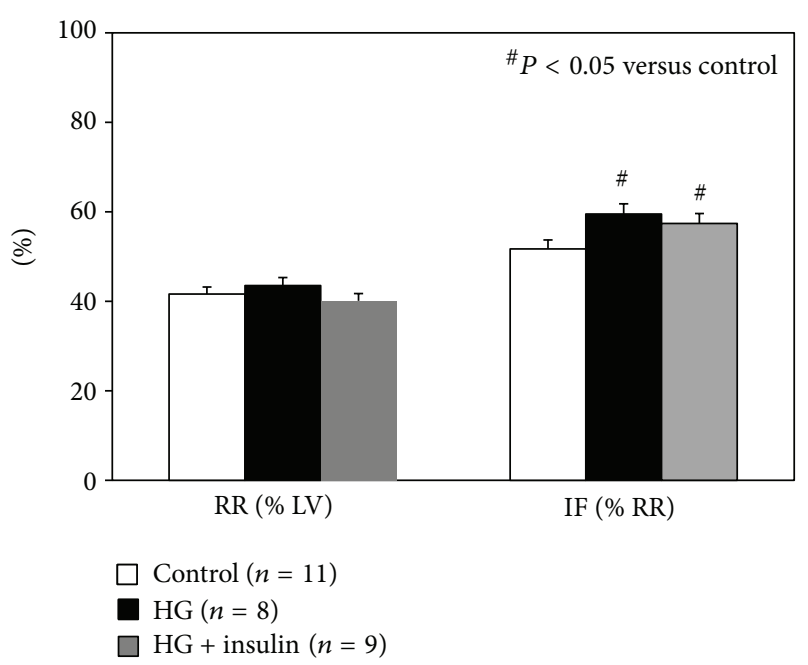

FIGURE 2: Myocardial infarct size after 40 minutes of LAD occlusion and 60 minutes of reperfusion. Acute hyperglycemia exacerbates infarct size. Normalization of blood glucose levels before LAD occlusion failed to counteract the hyperglycemic effect.

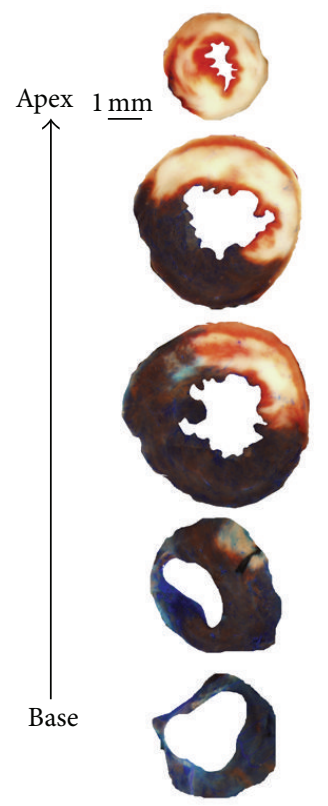

Control

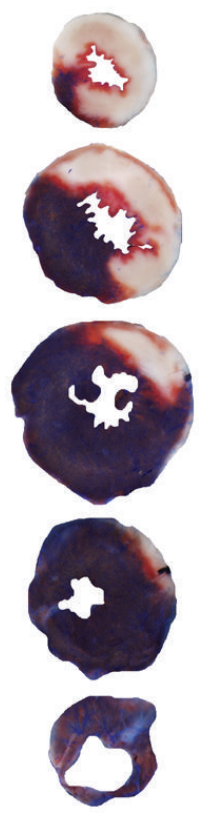

HG

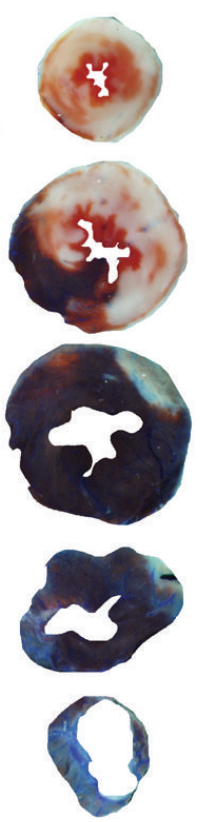

$\mathrm{HG}+$ insulin
FIGURE 3: TTC and Phthalo blue staining of representative hearts from groups corresponding to Figure 2.

to $59.5 \pm 2.3$ (a $15 \%$ increase, $P<0.05$ versus control). Infarct size was similarly enhanced both in $\mathrm{HG}+$ insulin mice and in HG mice $(57.4 \pm 2.2, P<0.05$ versus control, Figures 2 and 3).

3.4. Acute Hyperglycemia Abrogates the Effect of Ischemic Preconditioning and Insulin Restores It. As shown in Table 1, blood glucose levels before ischemic preconditioning or index ischemia were comparable among euglycemic and

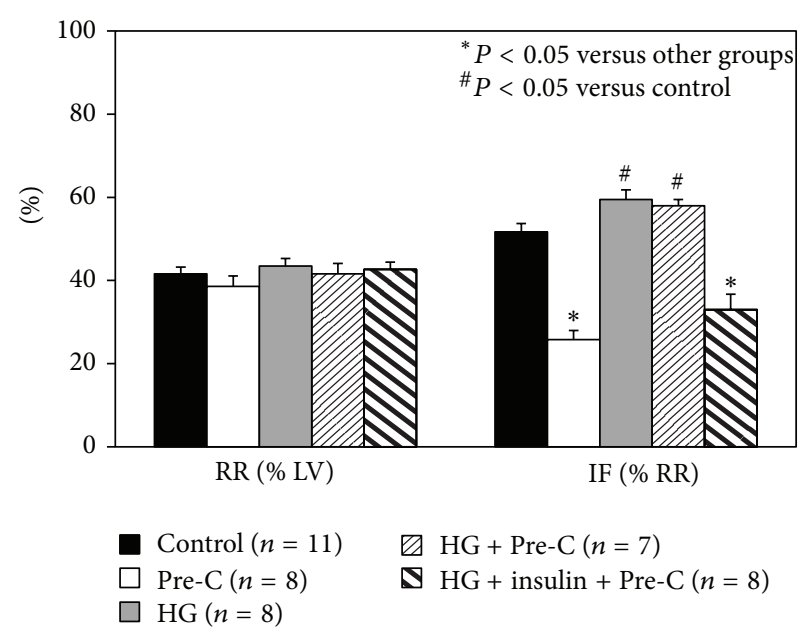

FIGURE 4: The cardioprotective effect of ischemic preconditioning disappears in HG mice, but can be recovered by normalizing blood glucose levels with insulin prior to ischemia.

hyperglycemic groups. Insulin treatment returned glucose levels back to the euglycemic baseline. There were no statistical differences in risk region ( $\mathrm{RR}, \%$ of $\mathrm{LV})$ among groups $(P>0.05$, left columns in Figure 4$)$. Ischemic preconditioning reduced infarct size to $25.8 \pm 3.2 \%$ (a 50\% reduction, $P<$ 0.05 versus control). This infarct-limiting effect completely disappeared in $\mathrm{HG}+$ Pre-C mice $(58.0 \pm 1.5 \%, P=\mathrm{NS}$ versus HG mice). Normalization of blood glucose with insulin $5 \mathrm{~min}$ before the preconditioning procedure served to partially restore the protective effect of preconditioning against myocardial infarction $(33.0 \pm 3.7 \%, P<0.05$ versus control or HG mice). Hearts from parallel groups of mice were harvested before the index $40 \mathrm{~min}$ LAD occlusion. The ratio of phosphorylated Akt relative to total Akt in heart tissue was found to be significantly enhanced in Pre-C mice but significantly inhibited in $\mathrm{HG}$ mice and $\mathrm{HG}+$ Pre-C mice relative to sham controls. However, normalization of blood glucose with insulin before the preconditioning protocol reversed the inhibitory effect of HG on Akt phosphorylation (Figure 5).

\subsection{Acute Hyperglycemia Abrogates the Cardioprotective Effect} of Adenosine $A_{1}$ Receptor Agonist. CCPA is a selective $\mathrm{A}_{1} \mathrm{R}$ agonist and induces a cardioprotective effect if applied before index ischemia. Preliminary dose response studies with CCPT revealed severe bradycardia in euglycemic mice at the dose of $100 \mu \mathrm{g} / \mathrm{kg}$ as well as $50 \mu \mathrm{g} / \mathrm{kg}$, which compromised survival during ischemia/reperfusion. Moreover, HG mice could not tolerate CCPA at these two doses and died during ischemia. A dose of CCPA at $25 \mu \mathrm{g} / \mathrm{kg}$ was therefore selected for the current study. Although this dose is significantly lower than those reported in the literature for other animal models, significant bradycardia occurred around $30 \mathrm{sec}$ after intravenous injection and then slowly improved. In euglycemic mice, CCPA decreased the heart rate from $413 \pm 17$ to $310 \pm 23$ beats/min (a $25 \%$ reduction, $P<0.05$ ). The heart rate slowly increased back to the baseline before index ischemia. Heart 


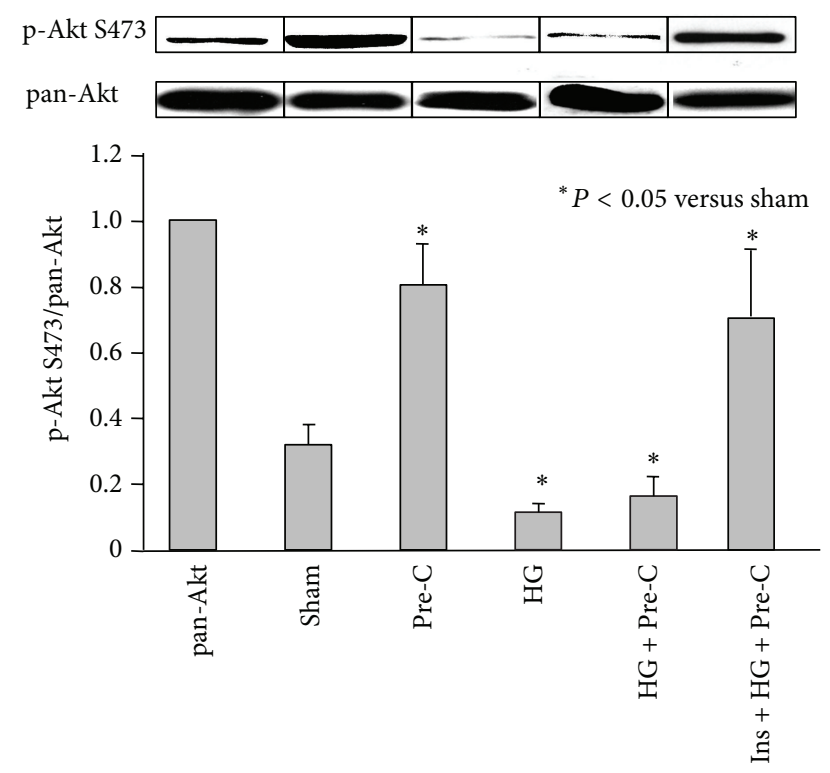

FIGURE 5: Myocardial phospho-Akt S473 to pan-Akt ratios in the indicated experimental groups. The ratio of phospho-Akt S473 to pan-Akt (the bar graph) was measured by densitometry, where the pan-AKT inputs were normalized to 1 .

rate in $\mathrm{HG}$ mice at baseline was not different than that in control mice, whereas CCPA decreased heart rate from $401 \pm$ 14 to $258 \pm 21$ beats $/ \mathrm{min}$ (a $36 \%$ reduction, $P<0.05$ ). Heart rate in HG mice increased to $345 \pm 15$ before index ischemia, which remained significantly lower than that at baseline $(P<$ $0.05)$. Glucose levels before LAD occlusion were $168 \pm 27$ in control mice, $186 \pm 8$ in CCPA-treated mice, and $355 \pm$ $23 \mathrm{mg} / \mathrm{dL}$ in CCPA-treated HG mice (both $P<0.05$ versus control). In CCPA-treated mice, infarct size was comparable to that of the Pre-C group ( $19.0 \pm 2.8$ versus $25.8 \pm 3.2, \%$ of $\mathrm{RR})$ and significantly smaller than that of control mice $(19.0 \pm$ 2.8 versus $51.7 \pm 2.0, P<0.05)$. However, the infarct-sparing effect of CCPA disappeared in HG mice (55.0 $\pm 4.2, P=\mathrm{NS}$ versus control; see Figure 6). Hearts from parallel groups of mice were harvested before the $40 \mathrm{~min}$ LAD occlusion. CCPA increased the ratio of phosphorylated to total Akt in heart tissue in euglycemic control mice by over 2 -fold $(P<0.05)$ but not in HG mice (Figure 7).

\section{Discussion}

An increasing body of evidence has shown that acute (or stress) hyperglycemia is an independent predictor of cardiovascular morbidity and mortality [3, 27-29]. Acute or stress hyperglycemia is associated with increased oxidative stress [8, 30-33], inflammation [34-37], and activation of stress-responsive kinase signaling [29, 35]. Infarcts are usually larger in patients with stress or diabetes-related hyperglycemia $[4,5,29,38]$, and animals with acute hyperglycemia sustain markedly larger infarcts following experimental ischemia/reperfusion than do euglycemic controls $[7,8,10,11]$. Moreover, acute hyperglycemia completely

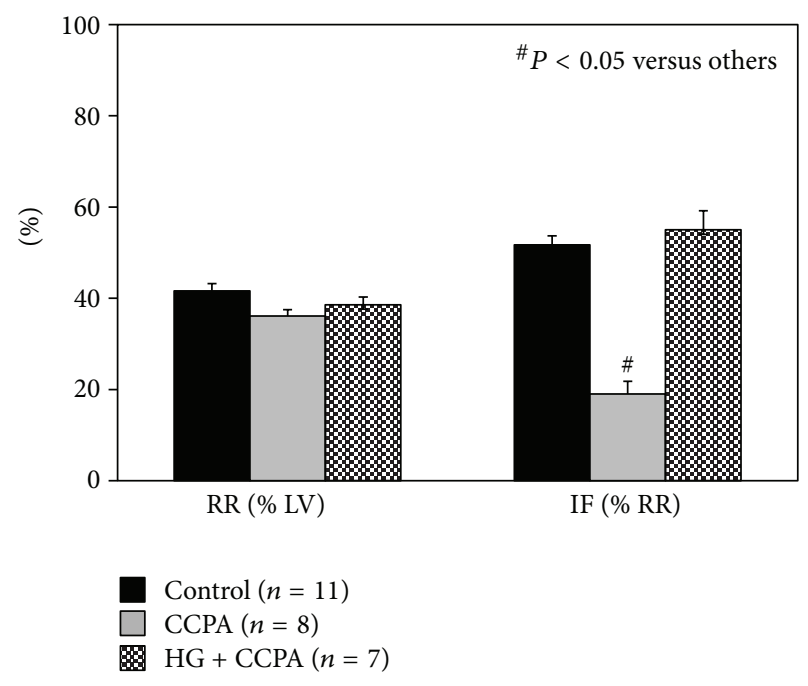

FIGURE 6: The cardioprotective effect of CCPA mimics the effect of ischemic preconditioning but disappears in HG mice.

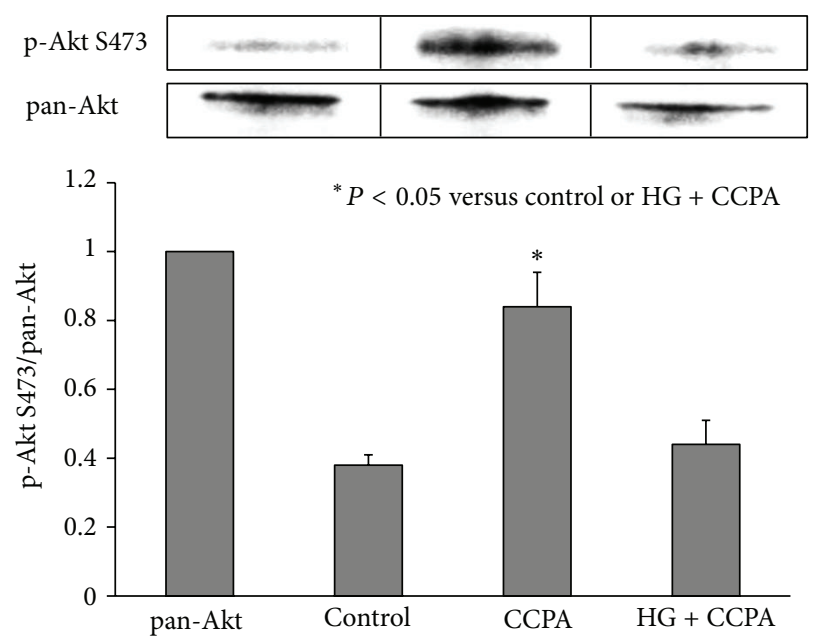

FIGURE 7: Myocardial phospho-Akt S473 to pan-Akt ratios in mice treated with CCPA.

abolishes the cardioprotective effect of ischemic preconditioning, negating a powerful endogenous cardioprotective mechanism and making cardiomyocytes more vulnerable to ischemia/reperfusion injury $[7,9,10,39,40]$. Pharmacological preconditioning has been reported to be inhibited by acute hyperglycemia as well $[7,15,39,41]$. The mechanisms underlying the hyperglycemic blockade of ischemic preconditioning remain unclear. Several lines of evidence point to 3 possible targets on which hyperglycemia may act to abolish the preconditioning effect: (1) increased production of reactive oxygen species [41, 42], (2) inhibition of PI3-Akt pathway [43, 44], and (3) inhibition of $\mathrm{K}_{\mathrm{ATP}}$ channels [17, 45]. However, MAP kinase appears not to be affected by the acute hyperglycemic event [12, 39].

Hyperglycemia negates the protective effect of ischemic preconditioning and, most importantly, appears to interfere with the salutary effects of insulin $[7,15]$. However, other 
studies have reported that administration of insulin may restore ischemic or pharmacological preconditioning [16, 17]. Insulin itself is a preconditioning-mimetic and exerts its salutary effect by activating the PI3-Akt pathway [13, $44,46]$. Clinically, aggressive therapy with insulin seems to improve a host of metabolic and physiologic effects associated with acute hyperglycemia and appears to be warranted if euglycemia cannot otherwise be maintained. However, an increasing body of clinical evidence has shown that acute hyperglycemia (or stress hyperglycemia) is independently associated with larger myocardial infarct size and impaired left ventricular function in both DM and nondiabetic patients $[4,5]$. The potential of insulin to mitigate against this hyperglycemic effect on myocardial ischemia/reperfusion injury is currently unclear, although clinical trials of glucose-insulinpotassium (GIK) therapy for acute myocardial infarction are certainly relevant. GIK therapy has been shown to improve cardiovascular performance after coronary artery surgery [2]. Moreover, a recent clinical trial on using early intravenous administration of GIK in patients with suspected acute coronary syndromes reported a significant decrease in the composite endpoint of cardiac arrest or in-hospital mortality (4.4\% in treated versus $8.7 \%$ in placebo group) [47]. Thus while GIK therapy was originally designed to provide balanced metabolic support to ischemic cardiomyocytes, it is possible that the beneficial effects of such a hyperinsulinemic/normoglycemic clamp might also involve the mechanisms investigated in the current study. This study employed a well-established in vivo mouse model to explore the role of insulin in treating acute myocardial ischemia/reperfusion injury and in restoring the effects of ischemic preconditioning during acute hyperglycemia.

In the current study, all the mice underwent 40 min LAD occlusion and $60 \mathrm{~min}$ reperfusion. The $40 \mathrm{~min}$ duration of LAD occlusion was selected to provide maximum sensitivity to both the detrimental effects of acute hyperglycemia and the cardioprotective effects of Pre-C. Longer LAD occlusions would reduce our sensitivity to detect infarct exacerbation due to hyperglycemia, while shorter occlusions would reduce our sensitivity to detect cardioprotection. The selection of 60 min of reperfusion is based on our previous study showing that myocardial infarction in mice attains $>95 \%$ of its final (24 hr) size within $60 \mathrm{~min}$ of reperfusion [48]. Acute hyperglycemia was demonstrated again to exacerbate myocardial infarct size as previously reported in a mouse model with $30 \mathrm{~min}$ LAD occlusion and $60 \mathrm{~min}$ reperfusion [8]. Normalization of blood glucose levels before the onset of ischemia failed to offset the infarct exacerbation secondary to the brief episode of acute hyperglycemia (Figure 2). Our previous study showed that acute hyperglycemia enhances oxidative stress and exacerbates myocardial infarct size in mice through the activation of NADPH oxidase [8]. Although insulin is cardioprotective via a preconditioningmimetic effect $[13,44,46]$, its salutary effect in the setting of acute hyperglycemia disappeared (Figure 2). The infarct exaggerating effect of acute hyperglycemia was retained even when the blood glucose level was normalized before the onset of ischemia, indicating that once hyperglycemia triggers pro-inflammatory signaling pathway [8], reversal of the hyperglycemia by insulin alone could not block the ongoing signal transduction. Acute hyperglycemia also makes cardiomyocytes vulnerable to injury by inhibiting the phosphorylation of Akt (Figure 5). However, the cardioprotective effect of insulin may be overshadowed by the detrimental effect of hyperglycemia.

In the ischemic preconditioning study, our results supported previous studies showing that acute hyperglycemia completely abolished the effect of ischemic preconditioning against myocardial ischemia/reperfusion injury $[7,9,10$, 39, 40]. Hyperglycemia exerted this effect by inhibiting the phosphorylation of Akt (Figures 4 and 5). To the best of our knowledge, this is the first report to show that the hyperglycemic loss of ischemic preconditioning against myocardial infarction is associated with an inhibition in the phosphorylation of Akt. Contrary to other reports [7, 15], our study showed that normalization of blood glucose before the preconditioning protocol completely recovered the cardioprotective effects of ischemic preconditioning (Figure 4). By normalizing blood glucose, insulin helped to restore the phosphorylation of Akt by ischemic preconditioning. Akt is an important mediator of cell survival and has long been implicated in ischemic preconditioning [49]. Insulin can activate the PI3K-Akt pathway and counteract oxidative stress, likely by increasing NO release [50-52]. The mechanisms underlying hyperglycemic inhibition of Akt phosphorylation, whether direct hyperglycemic effect or indirect, were not investigated in this study. We found that insulin has no direct cardioprotective effect in the setting of acute hyperglycemia but can recover the ischemic preconditioning effect to limit myocardial infarction. This phenomenon further confirmed our conclusion that acute hyperglycemia activates inflammatory responses and on the other hand decreases the internal defense mechanisms in cardiomyocytes.

The effect of hyperglycemia on preconditioning is most likely a direct effect of glucose on cardiomyocytes. Hyperglycemia has no direct effect in blocking adenosine $\mathrm{A}_{1}$ receptor $\left(A_{1} R\right)$ activation, which is a well-characterized mediator of ischemic preconditioning. Our data shows that acute hyperglycemia blocks ischemic preconditioning by disrupting signaling pathways downstream of $A_{1} R$ but not $A_{1} R$ activation itself since hyperglycemia blocked the cardioprotective effect of CCPA (Figures 6 and 7) but not the bradycardia caused by CCPA.

The current study provides indirect evidence to indicate that the inhibition of Akt phosphorylation by acute hyperglycemia is responsible for abolishing the cardioprotective effects of ischemic preconditioning. Nevertheless, these results clearly warrant future investigation into how acute hyperglycemia inhibits the phosphorylation of Akt, as well as the signal transduction pathways lying downstream of Akt. Future studies employing genetically manipulated mice and siRNA-mediated knockdowns are anticipated to further elucidate the effects of acute hyperglycemia on this clinicallyrelevant signal transduction pathway.

In conclusion, the current study clearly demonstrates that acute hyperglycemia exacerbates myocardial ischemia/ reperfusion injury and completely abolishes the cardioprotective effect of ischemic preconditioning by inhibiting Akt 
phosphorylation. Insulin treatment to normalize blood glucose levels failed to counteract the detrimental effect of hyperglycemia but could nevertheless restore the cardioprotective effects of ischemic preconditioning.

\section{Funding}

This study was funded by NIH R01 HL 092305 to Irving L. Kron and Brent A. French.

\section{References}

[1] H. L. Lazar, "Hyperglycemia during cardiac surgery," Journal of Thoracic and Cardiovascular Surgery, vol. 131, no. 1, pp. 11-13, 2006.

[2] D. W. Quinn, D. Pagano, R. S. Bonser et al., "Improved myocardial protection during coronary artery surgery with glucoseinsulin-potassium: a randomized controlled trial," Journal of Thoracic and Cardiovascular Surgery, vol. 131, no. 1, pp. 34.e242.e2, 2006.

[3] T. Doenst, D. Wijeysundera, K. Karkouti et al., "Hyperglycemia during cardiopulmonary bypass is an independent risk factor for mortality in patients undergoing cardiac surgery," Journal of Thoracic and Cardiovascular Surgery, vol. 130, no. 4, pp. 1144.e11144.e8, 2005.

[4] M. Ishihara, I. Inoue, T. Kawagoe et al., "Impact of acute hyperglycemia on left ventricular function after reperfusion therapy in patients with a first anterior wall acute myocardial infarction," American Heart Journal, vol. 146, no. 4, pp. 674-678, 2003.

[5] R. Marfella, M. Siniscalchi, K. Esposito et al., "Effects of stress hyperglycemia on acute myocardial infarction: role of inflammatory immune process in functional cardiac outcome," Diabetes Care, vol. 26, no. 11, pp. 3129-3135, 2003.

[6] N. N. Wahab, E. A. Cowden, N. J. Pearce, M. J. Gardner, H. Merry, and J. L. Cox, "Is blood glucose an independent predictor of mortality in acute myocardial infarction in the thrombolytic era?" Journal of the American College of Cardiology, vol. 40, no. 10, pp. 1748-1754, 2002.

[7] J. R. Kersten, W. G. Toller, E. R. Gross, P. S. Pagel, and D. C. Warltier, "Diabetes abolishes ischemic preconditioning: role of glucose, insulin, and osmolality," American Journal of Physiology-Heart and Circulatory Physiology, vol. 278, no. 4, pp. H1218-H1224, 2000.

[8] Z. Yang, V. E. Laubach, B. A. French, and I. L. Kron, "Acute hyperglycemia enhances oxidative stress and exacerbates myocardial infarction by activating nicotinamide adenine dinucleotide phosphate oxidase during reperfusion," Journal of Thoracic and Cardiovascular Surgery, vol. 137, no. 3, pp. 723-729, 2009.

[9] L. H. Opie, "Preconditioning and metabolic anti-ischaemic agents," European Heart Journal, vol. 24, no. 20, pp. 1854-1856, 2003.

[10] J. R. Kersten, "Acute hyperglycemia abolishes ischemic preconditioning in vivo," American Journal of Physiology-Heart and Circulatory Physiology, vol. 275, no. 2, pp. H721-H725, 1998.

[11] J. R. Kersten, M. W. Montgomery, T. Ghassemi et al., "Diabetes and hyperglycemia impair activation of mitochondrial KATP channels," American Journal of Physiology-Heart and Circulatory Physiology, vol. 280, no. 4, pp. H1744-H1750, 2001.

[12] D. Ebel, O. Toma, S. Appler et al., "Ischemic preconditioning phosphorylates mitogen-activated kinases and heat shock protein 27 in the diabetic rat heart," Hormone and Metabolic Research, vol. 41, no. 1, pp. 10-15, 2009.

[13] C. J. Zuurbier, "Insulin as ischaemic preconditioning-mimetic," Acta Physiologica, vol. 195, no. 2, p. 203, 2009.

[14] C. J. Zuurbier, O. Eerbeek, and A. J. Meijer, "Ischemic preconditioning, insulin, and morphine all cause hexokinase redistribution," American Journal of Physiology - Heart and Circulatory Physiology, vol. 289, no. 1, pp. H496-H499, 2005.

[15] D. Ebel, J. Müllenheim, J. Fräßdorf et al., "Effect of acute hyperglycaemia and diabetes mellitus with and without short-term insulin treatment on myocardial ischaemic late preconditioning in the rabbit heart in vivo," Pflugers Archiv European Journal of Physiology, vol. 446, no. 2, pp. 175-182, 2003.

[16] S. Forlani, F. Tomai, R. De Paulis et al., "Preoperative shift from glibenclamide to insulin is cardioprotective in diabetic patients undergoing coronary artery bypass surgery," Journal of Cardiovascular Surgery, vol. 45, no. 2, pp. 117-122, 2004.

[17] H. F. Del Valle, E. C. Lascano, and J. A. Negroni, "Ischemic preconditioning protection against stunning in conscious diabetic sheep: role of glucose, insulin, sarcolemmal and mitochondrial KATP channels," Cardiovascular Research, vol. 55, no. 3, pp. 642-659, 2002.

[18] J. D. Mccully, Y. Toyoda, M. Uematsu, R. D. Stewart, and S. Levitsky, "Adenosine-enhanced ischemic preconditioning: adenosine receptor involvement during ischemia and reperfusion," American Journal of Physiology-Heart and Circulatory Physiology, vol. 280, no. 2, pp. H591-H602, 2001.

[19] Z.-Q. Zhao, K. Nakanishi, D. S. McGee, P. Tan, and J. VintenJohansen, "A1 Receptor mediated myocardial infarct size reduction by endogenous adenosine is exerted primarily during ischaemia," Cardiovascular Research, vol. 28, no. 2, pp. 270-279, 1994.

[20] Z. Yang, R. L. J. Cerniway, A. M. Byford, S. S. Berr, B. A. French, and G. Paul Matherne, "Cardiac overexpression of Al-adenosine receptor protects intact mice against myocardial infarction," American Journal of Physiology-Heart and Circulatory Physiology, vol. 282, no. 3, pp. H949-H955, 2002.

[21] H. Takano, R. Bolli, R. G. Black Jr. et al., "A1 or A3 adenosine receptors induce late preconditioning against infarction in conscious rabbits by different mechanisms," Circulation Research, vol. 88, no. 5, pp. 520-528, 2001.

[22] M. E. Reichelt, A. Shanu, L. Willems et al., "Endogenous adenosine selectively modulates oxidant stress via the A1 receptor in ischemic hearts," Antioxidants and Redox Signaling, vol. 11, no. 11, pp. 2641-2650, 2009.

[23] A. R. Lankford, J.-N. Yang, R. Rose’Meyer et al., "Effect of modulating cardiac Al adenosine receptor expression on protection with ischemic preconditioning," American Journal of Physiology-Heart and Circulatory Physiology, vol. 290, no. 4, pp. H1469-H1473, 2006.

[24] E. Giannella, H.-C. Mochmann, and R. Levi, "Ischemic preconditioning prevents the impairment of hypoxic coronary vasodilatation caused by ischemia/reperfusion: role of adenosine A1/A3 and bradykinin B2 receptor activation," Circulation Research, vol. 81, no. 3, pp. 415-422, 1997.

[25] Z. Yang, Y.-J. Day, M.-C. Toufektsian et al., "Infarct-sparing effect of A2A-adenosine receptor activation is due primarily to its action on lymphocytes," Circulation, vol. 111, no. 17, pp. 2190 2197, 2005.

[26] Z. Yang, Y.-J. Day, M.-C. Toufektsian et al., "Myocardial infarctsparing effect of adenosine A2A receptor activation is due to its 
action on $\mathrm{CD}^{+}$T lymphocytes," Circulation, vol. 114, no. 19, pp. 2056-2064, 2006.

[27] S. E. Capes, D. Hunt, K. Malmberg, and H. C. Gerstein, "Stress hyperglycaemia and increased risk of death after myocardial infarction in patients with and without diabetes: a systematic overview," The Lancet, vol. 355, no. 9206, pp. 773-778, 2000.

[28] M. Ishihara, S. Kojima, T. Sakamoto et al., "Acute hyperglycemia is associated with adverse outcome after acute myocardial infarction in the coronary intervention era," American Heart Journal, vol. 150, no. 4, pp. 814-820, 2005.

[29] K. A. Webster, "Stress hyperglycemia and enhanced sensitivity to myocardial infarction," Current Hypertension Reports, vol. 10, no. 1, pp. 78-84, 2008.

[30] Y. Hu, G. Block, E. P. Norkus, J. D. Morrow, M. Dietrich, and M. Hudes, "Relations of glycemic index and glycemic load with plasma oxidative stress markers," American Journal of Clinical Nutrition, vol. 84, no. 1, pp. 70-76, 2006.

[31] H. Kawano, T. Motoyama, O. Hirashima et al., "Hyperglycemia rapidly suppresses flow-mediated endothelium-dependent vasodilation of brachial artery," Journal of the American College of Cardiology, vol. 34, no. 1, pp. 146-154, 1999.

[32] P. Mohanty, W. Hamouda, R. Garg, A. Aljada, H. Ghanim, and P. Dandona, "Glucose challenge stimulates reactive oxygen species (ROS) generation by leucocytes," Journal of Clinical Endocrinology and Metabolism, vol. 85, no. 8, pp. 2970-2973, 2000.

[33] M. G. Rosca, T. G. Mustata, M. T. Kinter et al., "Glycation of mitochondrial proteins from diabetic rat kidney is associated with excess superoxide formation," American Journal of Physiology-Renal Physiology, vol. 289, no. 2, pp. F420-F430, 2005.

[34] D. Sudic, M. Razmara, M. Forslund, Q. Ji, P. Hjemdahl, and N. Li, "High glucose levels enhance platelet activation: involvement of multiple mechanisms," British Journal of Haematology, vol. 133, no. 3, pp. 315-322, 2006.

[35] A. Aljada, J. Friedman, H. Ghanim et al., "Glucose ingestion induces an increase in intranuclear nuclear factor $\kappa \mathrm{B}$, a fall in cellular inhibitor $\kappa \mathrm{B}$, and an increase in tumor necrosis factor $\alpha$ messenger RNA by mononuclear cells in healthy human subjects," Metabolism, vol. 55, no. 9, pp. 1177-1185, 2006.

[36] S. Dhindsa, D. Tripathy, P. Mohanty et al., "Differential effects of glucose and alcohol on reactive oxygen species generation and intranuclear nuclear factor- $\kappa \mathrm{B}$ in mononuclear cells," Metabolism, vol. 53, no. 3, pp. 330-334, 2004.

[37] Y. Iwasaki, M. Kambayashi, M. Asai, M. Yoshida, T. Nigawara, and K. Hashimoto, "High glucose alone, as well as in combination with proinflammatory cytokines, stimulates nuclear factor kappa-B-mediated transcription in hepatocytes in vitro," Journal of Diabetes and its Complications, vol. 21, no. 1, pp. 5662, 2007.

[38] S. W. Zarich and R. W. Nesto, "Implications and treatment of acute hyperglycemia in the setting of acute myocardial infarction," Circulation, vol. 115, no. 18, pp. e436-e439, 2007.

[39] N. C. Weber, C. Goletz, R. Huhn et al., "Blockade of anaestheticinduced preconditioning in the hyperglycaemic myocardium. The regulation of different mitogen-activated protein kinases," European Journal of Pharmacology, vol. 592, no. 1-3, pp. 48-54, 2008.

[40] M. Ishihara, I. Inoue, T. Kawagoe et al., "Effect of acute hyperglycemia on the ischemic preconditioning effect of prodromal angina pectoris in patients with a first anterior wall acute myocardial infarction," American Journal of Cardiology, vol. 92, no. 3, pp. 288-291, 2003.

[41] F. Kehl, J. G. Krolikowski, D. Weihrauch, P. S. Pagel, D. C. Warltier, and J. R. Kersten, "N-acetylcysteine restores isoflurane-induced preconditioning against myocardial infarction during hyperglycemia," Anesthesiology, vol. 98, no. 6, pp. 13841390, 2003.

[42] F. B. Stentz and A. E. Kitabchi, "Hyperglycemia-induced activation of human T-lymphocytes with de novo emergence of insulin receptors and generation of reactive oxygen species," Biochemical and Biophysical Research Communications, vol. 335, no. 2, pp. 491-495, 2005.

[43] S. Matsumoto, S. Cho, S. Tosaka et al., "Pharmacological preconditioning in type 2 diabetic rat hearts: the roles of mitochondrial atp-sensitive potassium channels and the phosphatidylinositol 3-kinase-akt pathway," Cardiovascular Drugs and Therapy, vol. 23, no. 4, pp. 263-270, 2009.

[44] A. Tsang, D. J. Hausenloy, M. M. Mocanu, R. D. Carr, and D. M. Yellon, "Preconditioning the diabetic heart: the importance of Akt phosphorylation," Diabetes, vol. 54, no. 8, pp. 2360-2364, 2005.

[45] H. F. del Valle, E. C. Lascano, J. A. Negroni, and A. J. Crottogini, "Absence of ischemic preconditioning protection in diabetic sheep hearts: role of sarcolemmal KATP channel dysfunction," Molecular and Cellular Biochemistry, vol. 249, no. 1-2, pp. 21-30, 2003.

[46] A. K. Jonassen, M. N. Sack, O. D. Mjøs, and D. M. Yellon, "Myocardial protection by insulin at reperfusion requires early administration and is mediated via Akt and p70s6 kinase cellsurvival signaling," Circulation Research, vol. 89, no. 12, pp. 11911198, 2001.

[47] H. P. Selker, J. R. Beshansky, P. R. Sheehan et al., "Out-ofhospital administration of intravenous glucose-insulin-potassium in patients with suspected acute coronary syndromes: the IMMEDIATE randomized controlled trial," Journal of the American Medical Association, vol. 307, pp. 1925-1933, 2012.

[48] Z. Yang, J. Linden, S. S. Berr, I. L. Kron, G. A. Beller, and B. A. French, "Timing of adenosine $2 \mathrm{~A}$ receptor stimulation relative to reperfusion has differential effects on infarct size and cardiac function as assessed in mice by MRI," American Journal of Physiology-Heart and Circulatory Physiology, vol. 295, no. 6, pp. H2328-H2335, 2008.

[49] X. Yang, M. V. Cohen, and J. M. Downey, "Mechanism of cardioprotection by early ischemic preconditioning," Cardiovascular Drugs and Therapy, vol. 24, no. 3, pp. 225-234, 2010.

[50] G. Zeng, F. H. Nystrom, L. V. Ravichandran et al., "Roles for insulin receptor, PI3-kinase, and Akt in insulin-signaling pathways related to production of nitric oxide in human vascular endothelial cells," Circulation, vol. 101, no. 13, pp. 1539-1545, 2000.

[51] X.-Q. Zeng, C.-M. Zhang, M.-L. Tong et al., "Knockdown of NYGGF4 increases glucose transport in $\mathrm{C} 2 \mathrm{C} 12$ mice skeletal myocytes by activation IRS-1/PI3K/AKT insulin pathway," Journal of Bioenergetics and Biomembranes, vol. 44, no. 3, pp. 351355, 2012.

[52] T. Chen, G. Ding, Z. Jin et al., "Insulin ameliorates miR-1induced injury in $\mathrm{H} 9 \mathrm{c} 2$ cells under oxidative stress via Akt activation," Molecular and Cellular Biochemistry, vol. 369, pp. 167-174, 2012. 


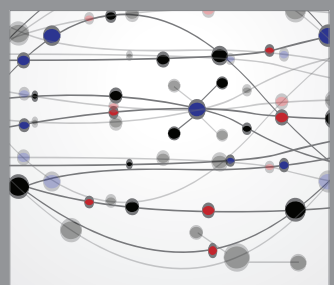

The Scientific World Journal
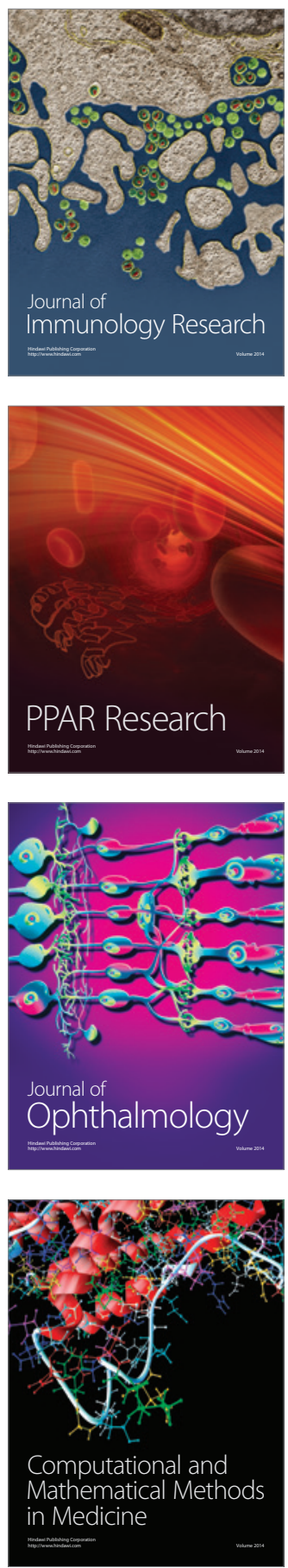

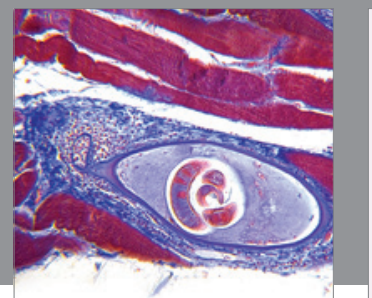

Gastroenterology

Research and Practice
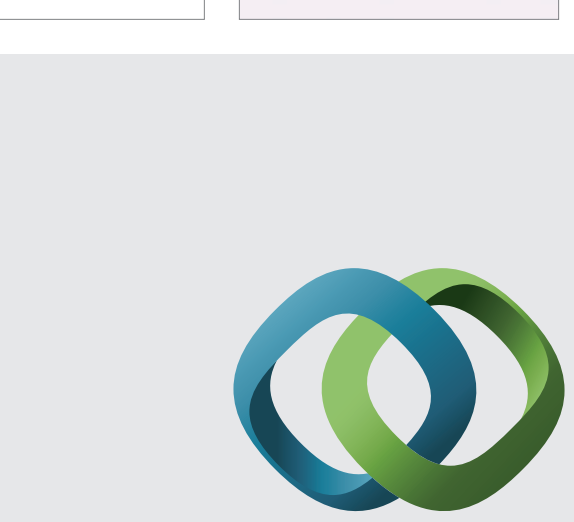

\section{Hindawi}

Submit your manuscripts at

http://www.hindawi.com
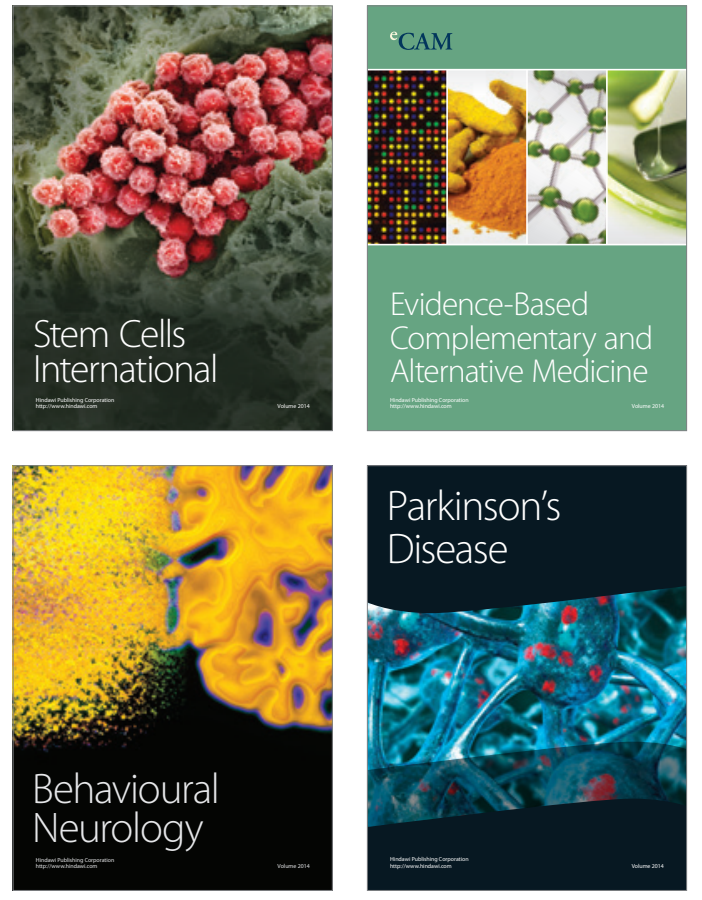
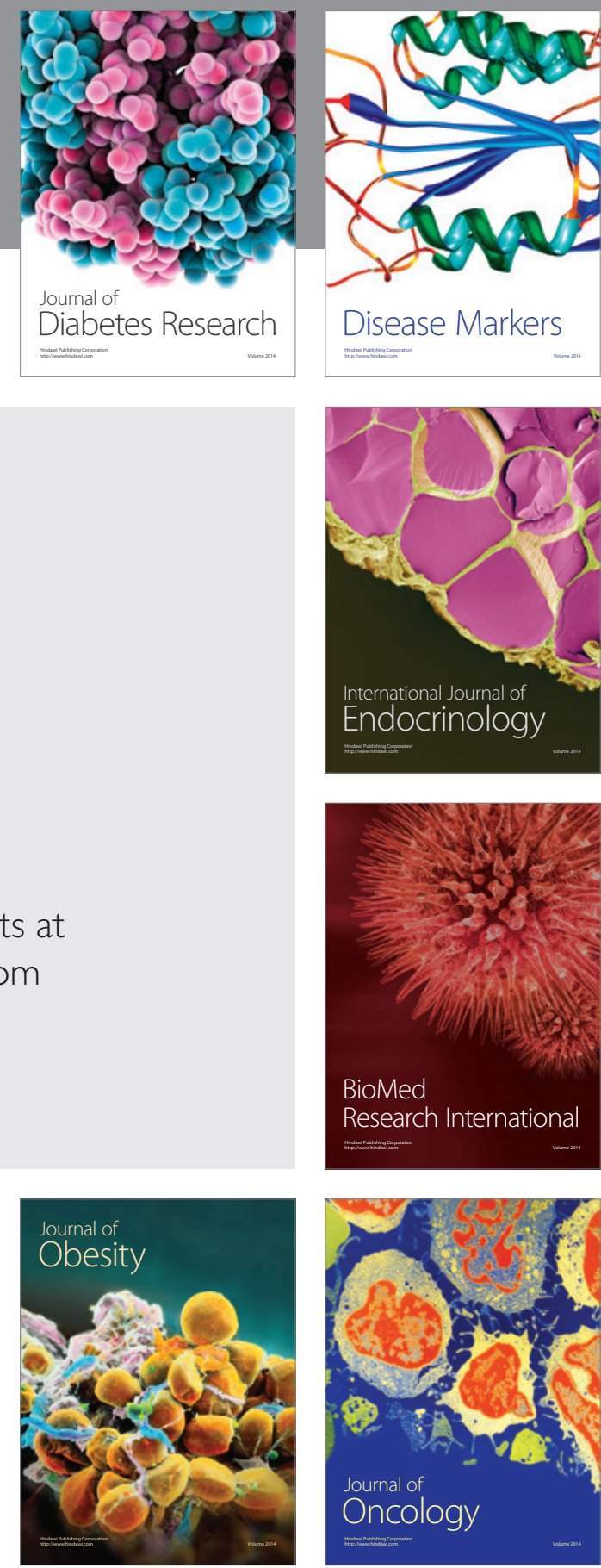

Disease Markers
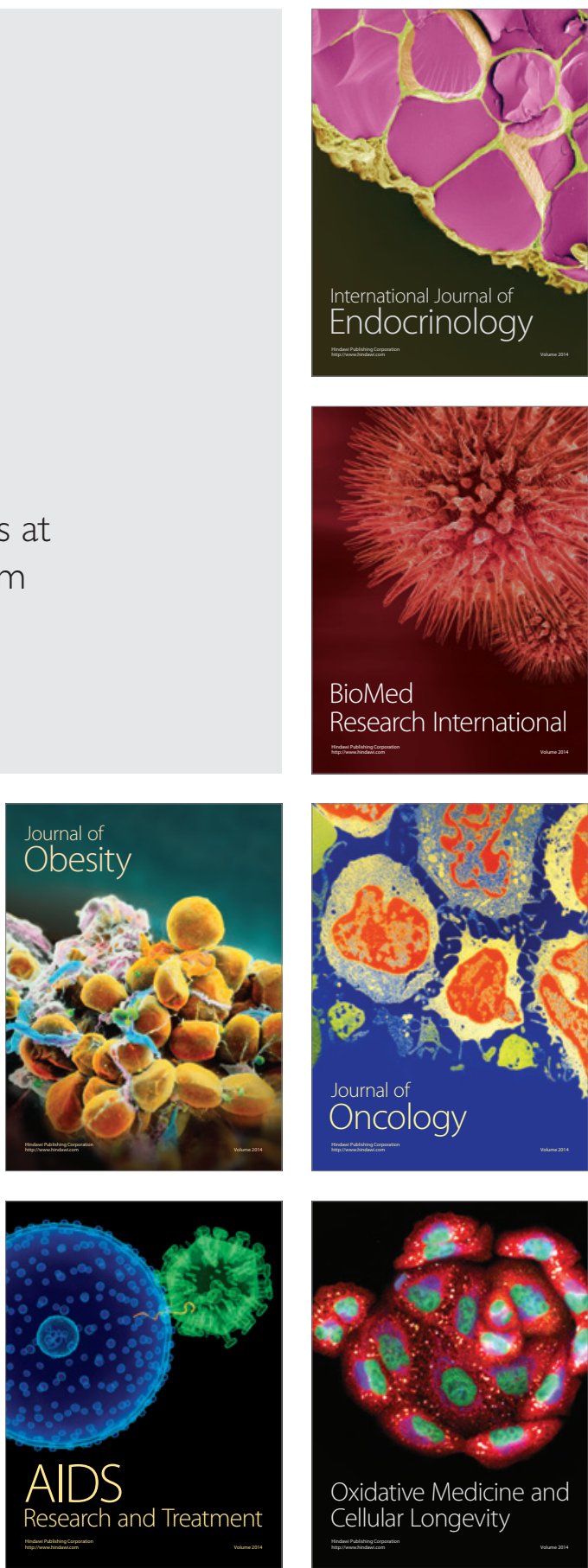